\title{
Clinical Importance of Diastolic Sonoelastographic Scoring in the Management of Thyroid Nodules
}

\author{
H. Yerli, T. Yilmaz, and I. Oztop
}

\begin{abstract}
SUMMARY: We determined that the application of a 5-point scoring method by using an external manual compression method synchronized with the diastolic period of the carotid artery by sonoelastography was helpful in the management of thyroid nodules. The mean scores on sonoelastography were $2.74 \pm 0.97$ for 62 benign nodules and $3.90 \pm 0.88$ for 10 malignant nodules $(P<.01)$. Eighty-seven percent of benign nodules were determined when a cutoff point between scores 3 and 4 was used.
\end{abstract}

ABBREVIATIONS: FNAB = fine-needle aspiration biopsy; SRU = Society of Radiologists in Ultrasound

B -mode sonography is usually the first imaging method for evaluating thyroid nodules. A significant disadvantage of Bmode sonography is its low specificity. Therefore, benign cytology is detected in most cases that have undergone FNAB on the basis of the findings of B-mode sonography. ${ }^{1}$

Sonoelastography is a sonography technique that directly reveals the elasticity features of tissue. The method determines tissue motion in response to externally or internally induced forces. When malignant lesions are compared with benign lesions, they are stiffer and less elastic. ${ }^{2,3}$ Several research groups used the natural vascular pulsation of the carotid artery as the internal compression source and determined a nodule strain index. ${ }^{4,5}$ Nevertheless, evaluation of nodules with this method is a laborious and time-consuming procedure not performed in real-time. Other researchers used the manual external compression method with a random phase approach on the thyroid region and scored the nodules. ${ }^{6-10}$ However, the quality of the sonoelastographic image might be worse in the random external manual compression method performed without considering the carotid artery systolic pulsation.

Unlike other researchers, we applied the manual external compression method in the diastole period. Therefore, we aimed to eliminate the deformation of the thyroid gland by the systolic

Received April 22, 2011; accepted May 19.

From the Departments of Radiology (H.Y.), General Surgery (T.Y.), and Pathology (I.O.), Baskent University Zubeyde Hanim, Practice and Research Center, Izmir, Turkey.

Please address correspondence to Hasan Yerli, MD, Department of Radiology, Baskent University Zubeyde Hanim, Practice and Research Center, 6371 Sokak No. 34 Bostanli/Karsiyaka, Izmir, Turkey, 35590; e-mail: hasanyerli@yahoo.com or hasyer@hotmail.com

http://dx.doi.org/10.3174/ajnr.A2751 pulsation of the carotid artery and to provide more effective compression. We scored the sonoelastographic images for thyroid nodules by slightly modifying the Tsukuba scoring method. ${ }^{11}$ Hence, our purpose was to determine whether the application of the elasticity scoring method by using manual compression synchronized with the diastolic period of the carotid artery by sonoelastography was helpful in the management of thyroid nodules.

\section{MATERIALS AND METHODS \\ Patients}

This prospective study was approved by the institutional review board of our university. Informed consent was obtained from all patients. Seventy-four thyroid nodules, for which surgery or FNAB was recommended, in 66 consecutive patients with normal pulse rates and blood pressure (age range, 20-87 years), were evaluated between October 2009 and June 2010. Two cases with inadequate aspiration material for diagnosis were excluded from the study. Therefore, the study group consisted of 59 women and 5 men, and 72 nodules were evaluated. Of the nodules included in the study, 58 fulfilled the FNAB-requiring criteria that the SRU defines. ${ }^{1}$ On the other hand, FNAB was recommended by endocrinologists for 14 solid nodules lacking the criteria defined by the SRU guideline because their largest diameter was $\geq 1 \mathrm{~cm}$. All patients were examined with B-mode sonography and sonoelastography. All the examinations were performed before surgery or FNAB.

\section{B-Mode Sonography and Sonoelastography}

All images were acquired by the EUB-7000 sonography system (Hitachi Medical Systems, Tokyo, Japan) including software with a combined autocorrelation method and a multihertz linear probe operating at 5-13 MHz. B-mode sonography and sonoelas- 


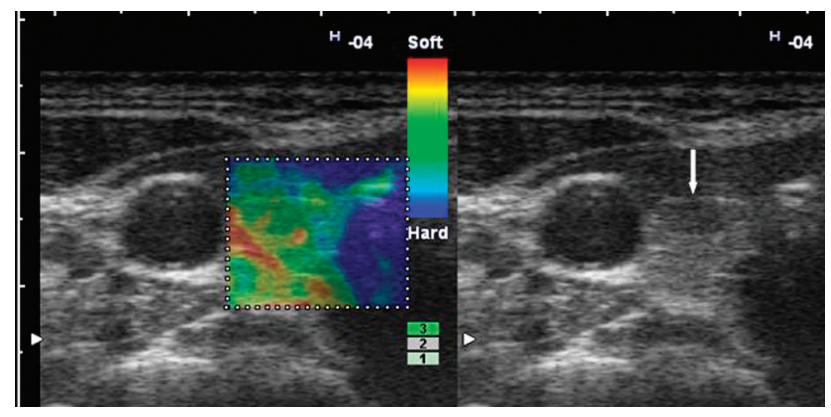

FIG 1. A benign nodule proved by FNAB. Right, B-mode sonography image shows a hypoechoic solid nodule (arrow). Left, Sonoelastographic image shows a lesion that is entirely green scored as 1.

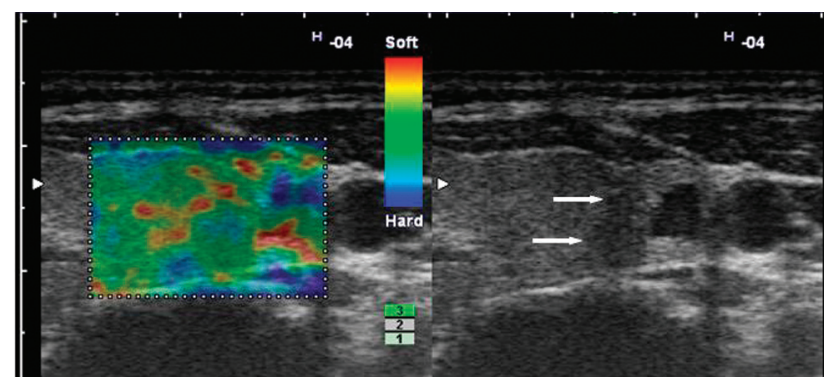

FIG 2. A benign nodule proved by FNAB. Right, B-mode sonography image shows a hypoechoic nodule taller than its width (arrows). Left, Sonoelastographic image shows a lesion that is almost entirely green with some blue points scored as 2 .

tography examinations were performed at the same session by 1 experienced radiologist with 10 years' experience in the field of thyroid sonography. All static and cine loop (moving images) images were digitally stored on the sonographic unit.

On the sonoelastographic examinations, when the screen was in real-time, sonoelastographic images together with B-mode sonography images were obtained by placing the transducer with coupling gel on the skin, activating the sonoelastography function with split-screen technology. The sonoelastographic images were color-coded as a map and superimposed on B-mode sonography in a translucent style on the left side and B-mode sonography images on the right side of the screen, to compare both images of the same scan plane. The region of interest mostly extended from the strap muscles to the posterior portion of the thyroid gland. The sonoelastographic images were obtained with appropriate compression. For this purpose, when we slightly pressed the probe in the diastole period, the pressure indicator bar in the middle of the screen showed 3 or 4 (Figs 1-5), and the vertical amplitude of probe was between 1 and $2 \mathrm{~mm}$. To manage the speed of probe movement, we used freehand management according to the diastole period of the common carotid artery on the screen during the external compressions because during the systole, higher blood pressure in the lumen of the carotid artery compresses the thyroid gland against the trachea in the transverse plane and causes thyroid gland expansion and deformation in the coronal plane. In our approach, the diastole phase of the carotid artery was determined visually from the sonography screen; therefore, external manual compression was synchronized with the diastolic period of the carotid artery. Care was taken to not

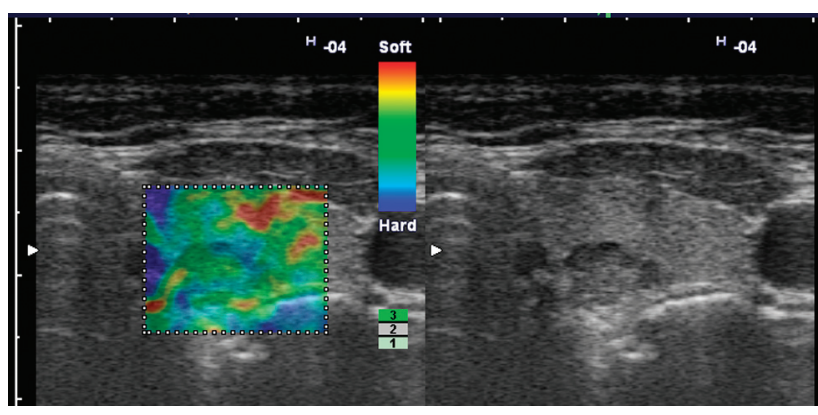

FIG 3. A benign nodule proved by histopathology. Right, B-mode sonography image shows a hypoechoic nodule with a microlobulated contour. Left, Sonoelastographic image shows a lesion that is predominantly green with some blue parts scored as 3 .

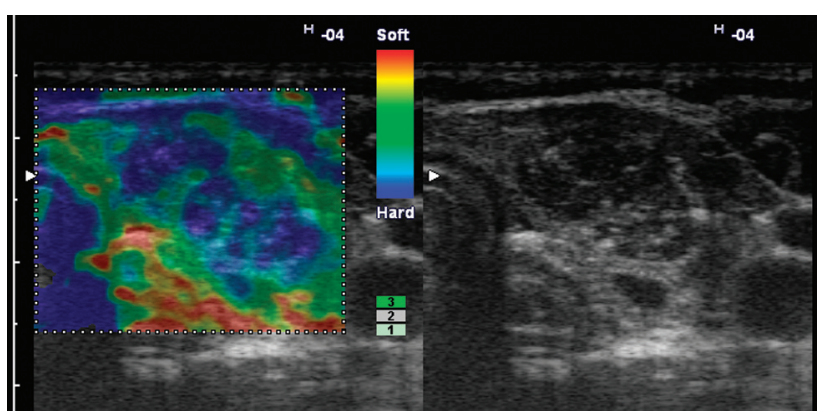

FIG 4. A papillary carcinoma proved by histopathology. Right, B-mode sonography image shows a hypoechoic nodule with microcalcifications. Left, Sonoelastographic image shows a lesion that is predominantly blue with some green parts scored as 4 .

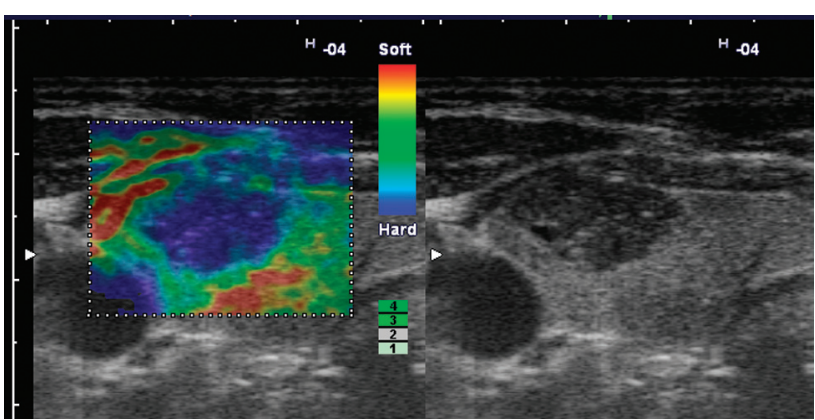

FIG 5. A papillary carcinoma proved by histopathology. Right, B-mode sonography image shows a hypoechoic nodule with microcalcifications in its central area. Left, Sonoelastographic image shows a lesion that is diffuse blue scored as 5 .

apply any external compression in the systolic phase of the carotid artery.

To obtain a correct sonoelastographic map, we repeated the process until a stable image was obtained. The sonoelastographic images were obtained according to a 256-color scale ranging from red to blue. The softest component of the nodule was demonstrated with red, showing the greatest strain, whereas the hardest component of the nodule, which did not strain, was demonstrated with blue. Green showed intermediate elasticity. In the evaluation of sonoelastographic images, nodule classification was performed on the basis of the 5-point scoring method (Table 1 and Figs 1-5).

To score the thyroid nodules, we slightly modified the scoring method, known as the Tsukuba scoring, that Itoh et $\mathrm{al}^{11}$ proposed for solid breast masses. In the Tsukuba classification, score 1 in- 
Table 1: The scores of sonoelastography modified from the Tsukuba scoring method for thyroid nodules

\begin{tabular}{lc}
\hline Score & Sonoelastographic Patterns for Each Score \\
\hline 1 & $\begin{array}{c}\text { Diffuse elastic pattern (homogeneously diffuse green) } \\
\text { An almost entirely elastic pattern (almost entirely green } \\
\text { with some blue points) }\end{array}$ \\
3 & $\begin{array}{c}\text { Mosaic of green and blue, but mostly elastic pattern } \\
\text { (mostly green) }\end{array}$ \\
4 & $\begin{array}{c}\text { Mosaic of green and blue, but most of the lesion had } \\
\text { no strain (mostly blue) }\end{array}$ \\
5 & $\begin{array}{c}\text { No strain (diffuse or almost entirely blue, and there } \\
\text { might also be a blue rim surrounding the lesion) }\end{array}$ \\
\hline
\end{tabular}

\begin{tabular}{|c|c|c|}
\hline Score Level & Benign $(n=62)(\%)$ & Malignant $(n=10)(\%)$ \\
\hline 1 & $9(14.5)$ & $0(0)$ \\
\hline 2 & $9(14.5)$ & $1(10)$ \\
\hline 3 & $36(58)$ & $1(10)$ \\
\hline 4 & $5(8)$ & $6(60)$ \\
\hline 5 & $3(5)$ & $2(20)$ \\
\hline
\end{tabular}

dicated strain for the whole lesion, score 2 indicated strain in most of the lesion with a mosaic pattern, score 3 indicated strain at the periphery of the lesion with a stiffness center, score 4 indicated stiffness in the whole lesion, and score 5 indicated no strain in the whole lesion and its surrounding area. Our classification that we modified differs from the Tsukuba scoring method mainly for the scores 3 and 4 . All sonoelastographic images for the classification of nodules were evaluated at a different session by 2 examiners in consensus without knowledge of the histopathologic diagnosis.

The final diagnoses of 10 malignant thyroid nodules and 14 benign nodules were determined by using FNAB and surgery. Thirty patients who had FNAB but not surgery were monitored by B-mode sonography follow-up every 6 months ( $>12$ months after FNAB). The diagnosis of 18 benign thyroid nodules was determined by using FNAB at least twice at a 1-year interval.

Differences among scores for benign and malignant thyroid nodules were assessed by using the Mann-Whitney $U$ test. Qualitative variables were compared by using the Pearson $\chi^{2}$ test. Twotailed $P<.05$ was considered statistically significant.

\section{RESULTS}

Of 72 thyroid nodules in the study, 62 were benign and 10 were malignant lesions. A hyperplastic nodule was observed in 14 of 24 nodules sent for histopathologic evaluation, while papillary carcinoma was observed in 10 of these. Some 48 nodules were diagnosed as benign on FNAB and follow-up.

The scores according to the 5-point scoring method on sonoelastography for benign and malignant nodules are seen in Table 2. The mean scores on sonoelastography were $2.74 \pm 0.97$ for benign nodules and $3.90 \pm 0.88$ for malignant nodules. The mean scores for malignant lesions were significantly higher than the corresponding values for benign lesions $(P<.01)$. The rate of score 4 was high in the cases of the malignant group, whereas the rate of score 3 was high in the cases of the benign group $(P<.01)$.

When a cutoff point between scores 3 and 4 was used, 8 falsepositives and 2 false-negatives were determined by the 5-point scoring system and $87 \%$ of benign nodules were diagnosed. The sensitivity, specificity, accuracy, positive predictive value, and negative predictive value were $83 \%, 89 \%, 88 \%, 56 \%$, and $97 \%$, respectively.

\section{DISCUSSION}

Elasticity is known as the spatial rate of change of displacement as a result of the tensile stress created by a certain amount of pressure on live tissue. A displacement in tissues can be created by external compression or internal physiologic forces, such as vascular pulsation. ${ }^{2,3}$ On the external compression method, strain evaluation by elastography in sonographic devices equipped with software is performed by periodic and short-term up and down movements of the probe on the tissue. During downward compression, slight pressure is applied. The strain level of the tissues can be observed in real-time in different color codes. In the systems involving the combined autocorrelation method developed by Shiina et $\mathrm{al},{ }^{12}$ which we also used in our study, there is a pressure scale simultaneously displaying the appropriateness of the manipulation as upward and downward movements on the sonography screen. The application is correct when the values displayed on the scale are 3 and 4 (Figs 1-5). This minimizes the variations on the basis of the operator during manipulation. ${ }^{11,12}$ Elasticity evaluation with the sonographic devices containing the combined autocorrelation method can be performed in several minutes. ${ }^{12}$

Dighe et $\mathrm{al}^{5}$ used the internal natural systolic pulsation of the carotid artery in the sonoelastographic examinations and determined a thyroid strain index that was computed as the ratio of strain near the main carotid artery to that of a low stiff region inside a thyroid nodule for the differential diagnosis of thyroid nodules. Lyshchik et $\mathrm{al}^{6}$ used the external manual compression method regardless of the systolic or diastolic period in the elasticity evaluation of thyroid nodules and scored nodules according to visualization, relative brightness, and margin regularity. By using a 4-point scale, they also calculated the strain index (thyroid gland-to-nodule ratio) of nodules. In both studies, off-line processing of strain images was considered laborious and time-consuming.

In the manual external compression method, the most important internal out-of-plane movement leading to artifacts is the systolic pulsation of the carotid artery when detecting the tissue elasticity occurring in the coronal plane. ${ }^{4,6}$ Unlike the methods applied in other studies, we performed manual probe compression by synchronizing it with the diastolic period of the main carotid artery. In our method, we aimed to eliminate the deformation occurring due to the compression effect created by the systolic pulsation on the transverse plane while compressing the thyroid gland, thereby performing more reliable and effective compression. In our study, we scored the thyroid nodules by slightly modifying the Tsukuba scoring method developed for solid breast masses. ${ }^{11}$

In study by Dighe et $\mathrm{al}^{5} 31(60.8 \%)$ of 51 benign nodules were detected before FNAB. In our study, FNAB was not needed for 54 $(87 \%)$ of 62 cases, for which FNAB had been recommended; our method had found the nodules benign. In some other studies performed by using manual compression and a combined autocorrelation method regardless of the systolic or diastolic period, ${ }^{8,10}$ and in our study, malignity was not detected in the nod- 
ules observed to have a homogeneous green color (score 1). Thus, we are of the opinion that the nodules included particularly in the category of score 1 may be reliably regarded as benign and excluded from the FNAB program. However, the disadvantage of our method is that it might be rather difficult to obtain a successful sonoelastography map in tachycardiac or tachypneic patients.

This study had limitations. First, the sonoelastography and B-mode sonography images were in the same scan plane due to the use of split-screen technology during the sonoelastographic evaluations. This evaluation form may cause an initial opinion based on B-mode sonography that might affect sonoelastographic comment. Second, we did not evaluate the interobserver variability when scoring. Although the equipment used in our study had technology reflecting the degree of compression pressure during sonoelastographic examinations, the extent of appropriate compression that might vary by examiner can influence the elasticity image.

In conclusion, the 5-point scoring method by using the external manual compression method synchronized with the diastolic period of the carotid artery by sonoelastography can potentially be helpful in the management of thyroid nodules.

\section{REFERENCES}

1. Frates MC, Benson CB, Charboneau JW, et al. Management of thyroid nodules detected at US: Society of Radiologists in Ultrasound consensus conference statement. Radiology 2005;237:794-800
2. Ophir J, Céspedes I, Ponnekanti H, et al. Elastography: a quantitative method for imaging the elasticity of biological tissues. Ultrason Imaging 1991;13:111-34

3. Garra $\mathrm{BH}$. Imaging and estimation of tissue elasticity by ultrasound. Ultrasound Q 2007;23:255-68

4. Bae U, Dighe M, Dubinsky T, et al. Ultrasound thyroid elastography using carotid artery pulsation: preliminary study. J Ultrasound Med 2007;26:797-805

5. Dighe M, Kim J, Luo S, et al. Utility of the ultrasound elastographic systolic thyroid stiffness index in reducing fine-needle aspirations. J Ultrasound Med 2010;29:565-74

6. Lyshchik A, Higashi T, Asato R, et al. Thyroid gland tumor diagnosis at US elastography. Radiology 2005;237:202-11

7. Rago T, Santini F, Scutari M, et al. Elastography: new developments in ultrasound for predicting malignancy in thyroid nodules. J Clin Endocrinol Metab 2007;92:2917-22

8. Asteria C, Giovanardi A, Pizzocaro A, et al. US-elastography in the differential diagnosis of benign and malignant thyroid nodules. Thyroid 2008;18:523-31

9. Tranquart F, Bleuzen A, Pierre-Renoult P, et al. Elastosonography of thyroid lesions. J Radiol 2008;89:35-39

10. Rubaltelli L, Corradin S, Dorigo A, et al. Differential diagnosis of benign and malignant thyroid nodules at elastosonography. Ultraschall Med 2009;30:175-79. Epub 2008 May 21

11. Itoh $\mathrm{A}$, Ueno $\mathrm{E}$, Tohno $\mathrm{E}$, et al. Breast disease: clinical application of US elastography for diagnosis. Radiology 2006;239:341-50

12. Shiina $T$, Nitta N, Ueno E, et al. Real time tissue elasticity imaging using the combined autocorrelation method. J Med Ultrasonics 2002;29:119-28 